\title{
Valence Band Splitting in Wurtzite InP Nanowires Observed by Photoluminescence and Photoluminescence Excitation Spectroscopy
}

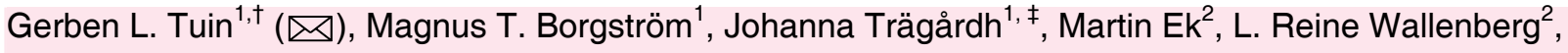 \\ Lars Samuelson ${ }^{1}$, and Mats-Erik Pistol ${ }^{1}(\bowtie)$ \\ ${ }^{1}$ Solid State Physics/Nanometer Structure Consortium, Box 118, Lund University, S-221 00 Lund, Sweden \\ ${ }^{2}$ Polymer \& Materials Chemistry/nCHREM, Lund University, S-22100 Lund, Sweden \\ ${ }^{\dagger}$ Work performed while on leave from: Kavli Institute of Nanoscience, Delft University of Technology, P.O. Box 5046, 2600 GA Delft \\ ${ }^{\star}$ Present address: H. H. Wills Physics Laboratory, University of Bristol, Tyndall Avenue, Bristol, BS8 1TL United Kingdom \\ Received: 23 August 2010 / Revised: 14 October 2010 / Accepted: 26 October 2010 \\ (C) Tsinghua University Press and Springer-Verlag Berlin Heidelberg 2010
}

\begin{abstract}
We have investigated individual bulk-like wires of wurtzite InP using photoluminescence, photoluminescence excitation spectroscopy and transmission electron microscopy. Using two different methods we find that the top of the valence band is split, as expected theoretically. This splitting of the valence band is peculiar to wurtzite InP and does not occur in zinc blende InP. We find the energy difference between the two bands to be $40 \mathrm{meV}$.
\end{abstract}

\section{KEYWORDS}

wurtzite InP, photoluminescence, excitation spectroscopy, valence band structure

Recently, the possibility of controlling the crystal phase in nanowires (NWs) - which opens up new opportunities for band structure engineering using only a single material-has received much interest. In particular, the crystal structure of InP NWs can be controlled by varying the growth parameters to produce either zinc blende (ZB) or wurtzite (WZ), as has been shown experimentally [1-7] and modelled theoretically [8-10]. The nanowire diameter $[4,5,7,9,10]$ in particular is correlated with the crystal structure, where a thinner wire tends to favour wurtzite formation. In addition, in situ nanowire doping has been found to strongly affect the crystal phase [4]. We observed that sulfur doping favours wurtzite structure formation. It is of great interest to characterise the mostly unknown bulk properties of these new types of materials.
Optical investigations at $4 \mathrm{~K}$ on wurtzite $\mathrm{InP}$ nanowires indicated a bandgap energy of $1.49-1.50 \mathrm{eV}[2,6,12]$ which is higher than the bandgap of zinc blende InP $(1.41 \mathrm{eV})$. In order to assess the bulk-like properties of wurtzite InP, we grew $15 \mathrm{~nm}$ sulfur-doped InP nanowire cores in the wurtzite phase, as confirmed by transmission electron microscopy. Bulk-like material was then created by growth of a thick intrinsic epitaxial InP shell around the nanowires. By "bulk-like" we mean that the wires were quite thick and no quantum confinement was present.

In this article we report photoluminescence (PL) and PL excitation (PLE) spectroscopy studies of such individual bulk-like wurtzite $\mathrm{InP}$ wires. We find that the bandgap energy is in agreement with previous studies. Figure 1 shows the band structure of wurtzite

Address correspondence to Mats-Erik Pistol, mats-erik.pistol@ftf.lth.se; Gerben L. Tuin, GLTuin@gmail.com 

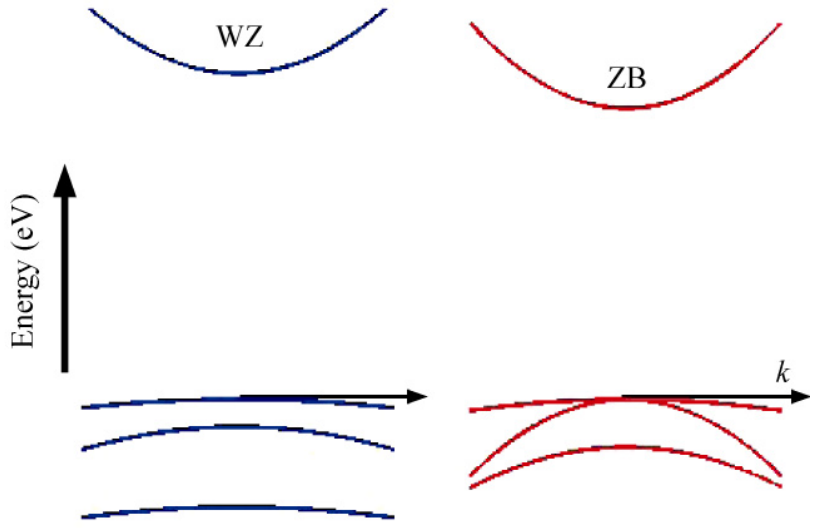

Figure $1 E-k$ diagrams for wurtzite (WZ) and zinc blende (ZB) InP. In wurtzite InP the top of the valence band is split into two bands, each of which is doubly degenerate (where spin is included). The spin-orbit split-off band is also included in the figure. The top of the valence band is fourfold degenerate for zinc blende InP

and zinc blende InP: note that the top of the valence band is split in wurtzite InP in contrast to zinc blende InP. We experimentally determine this splitting to be about $40 \mathrm{meV}$, which is in good agreement with theoretical calculations [11] and PLE experiments [12]. We find that we can populate the second valence band using a high excitation power density, which allows a fairly easy determination of the energy splitting, in comparison with previous work where PLE was needed to determine this parameter [12].

Samples for growth were prepared by depositing $15 \mathrm{~nm}$ aerosol particles on a (111)B InP substrate via an aerosol technique [13] resulting in randomly distributed particles with a homogeneous surface density of $5 \times 10^{7} / \mathrm{cm}^{2}$. We used low pressure (100 mbar) metal-organic vapour phase epitaxy (MOVPE) for particleassisted nanowire growth of the core. Trimethylindium (TMI), phosphine $\left(\mathrm{PH}_{3}\right)$ and hydrogen sulphide $\left(\mathrm{H}_{2} \mathrm{~S}\right)$ were used as precursors in a total flow of $6 \mathrm{~L} / \mathrm{min}$ using hydrogen as a carrier gas. $\mathrm{HCl}$ was used as an in situ etching agent in order to prevent any tapering. The $\mathrm{PH}_{3}$, TMI, and $\mathrm{H}_{2} \mathrm{~S}$ molar fractions $\left(\chi\left(\mathrm{PH}_{3}\right), \chi(\mathrm{TMI})\right.$, $\left.\chi\left(\mathrm{H}_{2} \mathrm{~S}\right)\right)$ were set to $\chi\left(\mathrm{PH}_{3}\right)=6.2 \times 10^{-3}, \chi(\mathrm{TMI})=3.5 \times 10^{-6}$ and $\chi\left(\mathrm{H}_{2} \mathrm{~S}\right)=7.1 \times 10^{-6}$ for growth of the wurtzite cores. The $\mathrm{HCl}$ molar fraction was set to $\chi(\mathrm{HCl})=8.3 \times 10^{-6}$. The samples were first heated to $550{ }^{\circ} \mathrm{C}$ under a $\mathrm{PH}_{3} / \mathrm{H}_{2}$ gas mixture for $10 \mathrm{~min}$ in order to desorb any surface oxides. The reactor temperature was then reduced to $420{ }^{\circ} \mathrm{C}$, at which temperature growth was initiated by adding TMI to the flow. $\mathrm{H}_{2} \mathrm{~S}$ and $\mathrm{HCl}$ were added simultaneously after a $15 \mathrm{~s}$ nucleation step. After growth of the core for $20 \mathrm{~min}, \mathrm{TMI}, \mathrm{H}_{2} \mathrm{~S}$, and $\mathrm{HCl}$ were switched off simultaneously, and the temperature ramped up to $600{ }^{\circ} \mathrm{C}$, at which point TMI was switched on to grow a non-intentionally doped epitaxial shell for $15 \mathrm{~min}$, resulting in wires with a diameter of $100 \mathrm{~nm}$. Samples were prepared for high-resolution transmission electron microscopy (HRTEM) by direct transfer of the wires to a lacey carbon coated copper grid by gently pressing the grid onto the substrate. The wires were characterised by HRTEM using a JEOL 3000F instrument operating at $300 \mathrm{kV}$. The wires were tilted into the $\langle 11 \overline{2} 0\rangle$ zone axis and imaged over their entire length.

For PL(E) measurements, the wires were deposited on a silicon substrate, covered with a gold layer sufficiently thick to suppress interference from the silicon photoluminescence. PL measurements were carried out using the $532 \mathrm{~nm}$ line from a frequencydoubled Nd-YAG laser, which was focused through a microscope objective. The signal was then collected through the same microscope objective, dispersed through a spectrometer and detected by a CCD-camera. For PLE and temperature-dependent measurements a CW Ti:sapphire laser was coupled in at a $45^{\circ}$ angle outside the microscope to create a large excitation area with an excitation power density of approximately $3 \mathrm{nW} / \mu \mathrm{m}^{2}$. A neutral density filter was used to control the laser power incident on the nanowires. Figure 2 shows a TEM image of a wurtzite InP core shell wire.

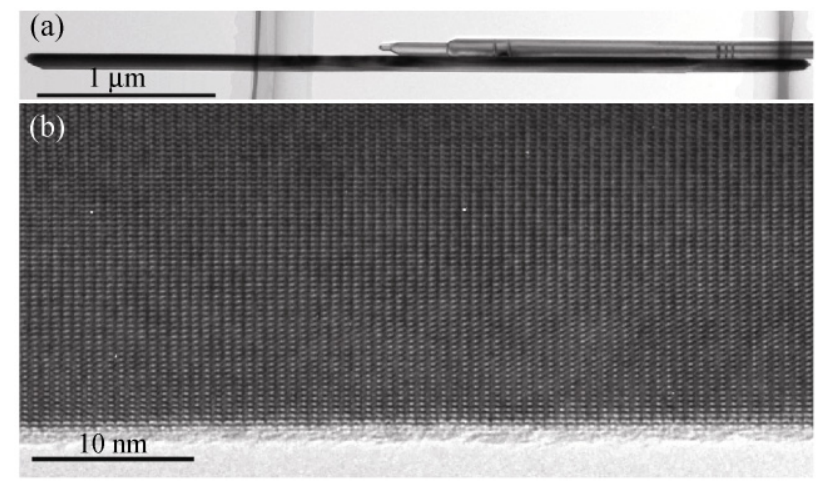

Figure 2 TEM images of InP wurtzite wires. (a) Overview image of one wire viewed along the $<11 \overline{2} 0>$ axis. An adjacent wire which is off axis is also shown. The dark bands in the latter indicate the presence of stacking faults and/or short zinc blende segments. (b) A high resolution image showing the pure wurtzite structure which constitutes the majority of the wire 
Long segments $(>1 \mu \mathrm{m})$ of pure wurtzite structure, which are interrupted by stacking faults or short zinc blende segments, are observed in the wires.

From TEM imaging, the core shell volume ratio is estimated to be 1:50. Sulfur is not expected to diffuse into the shell, due to its low diffusion constant [14]. Therefore, we argue that luminescence from the coreshell wires mainly originates from the large volume of the bulk-like, undoped, InP wurtzite shell. In Fig. 3 we show luminescence from a wire grown without a shell, which shows emission at a fairly high energy, $1.6 \mathrm{eV}$, representing quite a marked blue shift of the emission energy compared with undoped InP. This is due to the high density of donors. This blue shift is not seen in the wires grown with an undoped shell.

The PL spectra of two InP wurtzite wires, having an undoped shell outside the core are depicted in Fig. 4. The spectra were obtained at $4 \mathrm{~K}$ using a maximum excitation power density of $P=0.1 \mathrm{~mW} / \mu^{2}$. At a low excitation power density, $0.3-3 \mathrm{nW} / \mu \mathrm{m}^{2}$, two peaks can be seen. The higher energy peak at $1.493 \mathrm{eV}$ is attributed to transitions from the conduction band to the valence band, most likely involving excitons. We

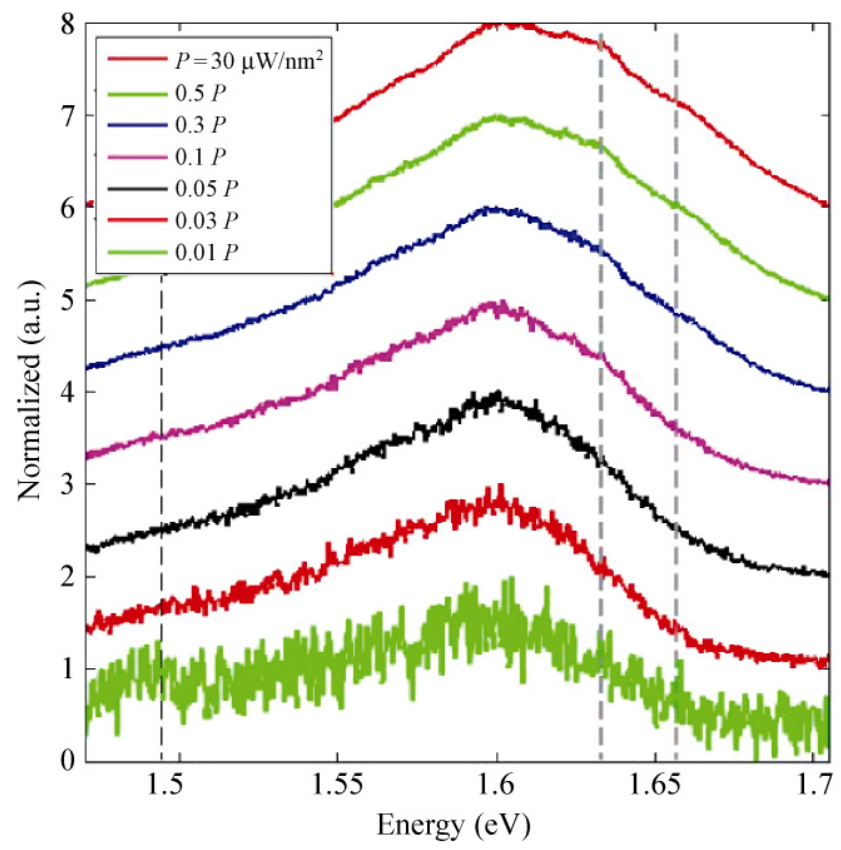

Figure 3 Photoluminescence spectra of a control sample, a wire without a shell, i.e., consisting only of the sulfur-doped core. Seven different excitation power densities were used, indicated in the figure. The emission peaks are at a considerably higher energy than that from undoped wurtzite InP

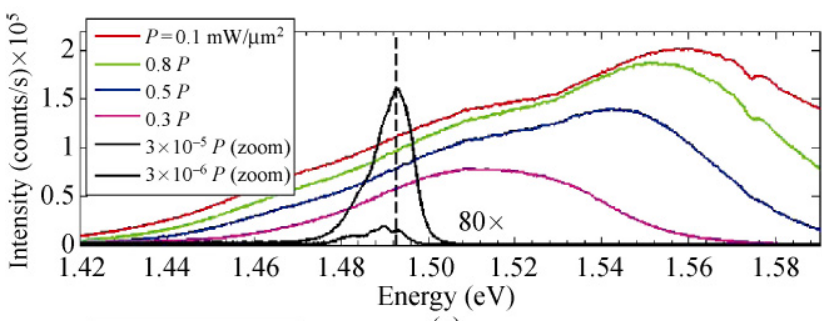

(a)

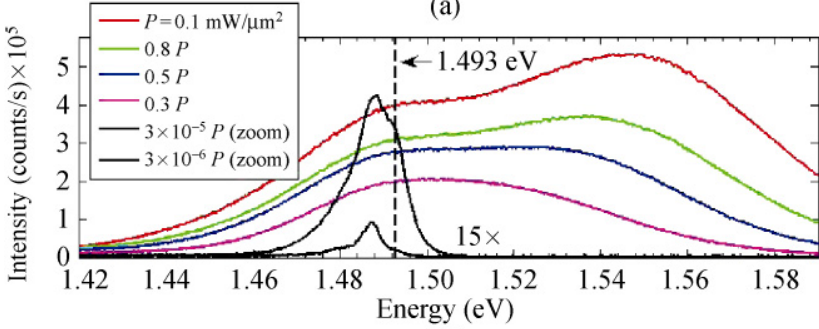

(b)

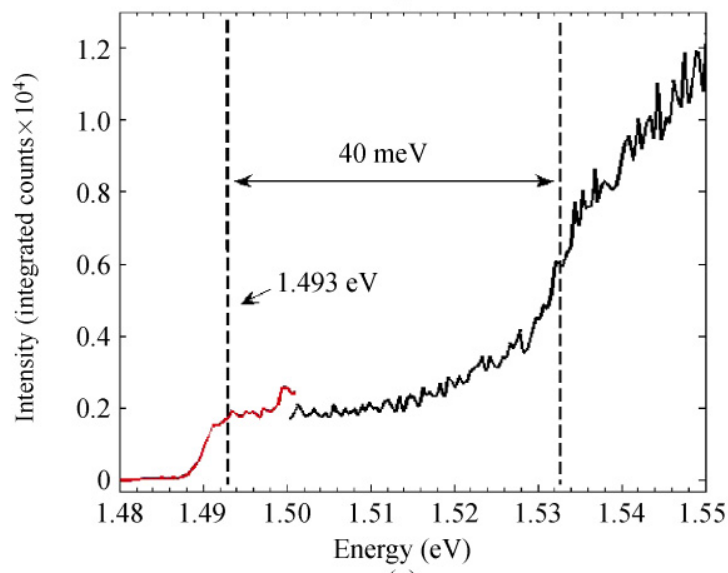

(c)

Figure 4 (a, b) PL spectra of two different wires at low excitation power densities (black traces), magnified for clarity (as stated on the right side of the traces), and high excitation power densities (coloured traces). The position of the exciton-related peak is indicated by the dashed line. (c) PLE spectrum of one bulk-like wurtzite InP wire. Red/black traces indicate separate measurements with detection at $1.48 \mathrm{eV}$ and at $1.5 \mathrm{eV}$. The dashed lines indicate transitions between the conduction band and the first and second valence bands based on the PL data. The second onset is about $40 \mathrm{meV}$ higher in energy than the bandgap energy

will refer to this peak as the exciton peak. The exciton binding energy is expected to be low, about $5 \mathrm{meV}$, and will not substantially affect our results with regards to, for example, the bandgap energy. Our bandgap energy is slightly lower than that measured in previous work [12]. Carrier-carrier interaction is known to reduce the bandgap in some cases [14], but will also destroy the exciton and give broad emission lines, in contrast to our observation. The core itself has a very different emission, see Fig. 3 . It is more likely that the 
small difference in the measured bandgap is due to different band bending in our case, compared with other samples [12]. Some influence of the surface, such as surface charges affecting the band bending, is expected in small wires. The transition at lower energy, $1.488 \mathrm{eV}$, is attributed to impurities and will be referred to as the impurity peak. We have indicated the bandgap energy by a dotted line. A large number of wires were characterised at an excitation power density of $3 \mathrm{nW} / \mu \mathrm{m}^{2}$ with similar results. No transitions at energies above $1.49 \mathrm{eV}$ were found in any of the wires, at these excitation power densities. The impurity peak varied in intensity relative to the exciton peak depending on the wire (compare Figs. 4(a) and 4(b)) and had an energy about $5-6 \mathrm{meV}$ below the exciton peak. At higher excitation power density, the exciton recombination eventually dominates over the impurity peak, as expected.

At even higher excitation power densities (Fig. 4, colored traces) a peak at higher energy emerges, at an energy around $1.56 \mathrm{eV}$. This peak, which we observe in every wire, we attribute to a transition between the conduction band and the second valence band (see Fig. 1). Band structure calculations by De and Pryor [11] find the split between the first and the second valence band to be about $60 \mathrm{meV}$ in wurtzite $\mathrm{InP}$, which is in reasonable agreement with our experimental value of about $40 \mathrm{meV}$. We attribute the tail on the low energy side, at high excitation power density, to bandgap renormalization $[2,15]$.

In order to strengthen our assignment of this peak to the second valence band, we performed low temperature PLE measurements, shown in Fig. 4. Detecting at $1.48 \mathrm{eV}$, we observed a PLE onset at an energy around $1.488 \mathrm{eV}$, which coincides with the impurityrelated peak. This onset levels out at an energy around the exciton-related peak, indicated by a dotted line at $1.493 \mathrm{eV}$. About $40 \mathrm{meV}$ higher, a second onset is visible when the detection is at the exciton peak. This second onset is precisely what is expected if the top of the valence band is split, and confirms our interpretation from the band-filling experiment.

The same energy splitting was also seen in room temperature PL spectra of four different wires (see Fig. 5). The second valence band recombination is indicated by a peak around $1.464 \mathrm{eV}$, approximately
$40 \mathrm{meV}$ higher than the bandgap at $1.427 \mathrm{eV}$.

In order to gain more insight into the exciton- and impurity-related peaks, temperature-dependent PL measurements were performed (Fig. 5). For these measurements a low excitation power density was used $\left(3 \mathrm{nW} / \mu \mathrm{m}^{2}\right)$ which results in well separated peaks. The activation energy of the impurity peak is estimated to be around $5 \mathrm{meV}$, which is close to the energy difference between the impurity peak and the exciton peak observed in photoluminescence. We find that the exciton peak has a relatively constant intensity up to about $40 \mathrm{~K}$ and then starts to quench. The total PL intensity decreases as we increase the temperature, and there must thus be non-radiative channels that become more prominent at higher temperatures.
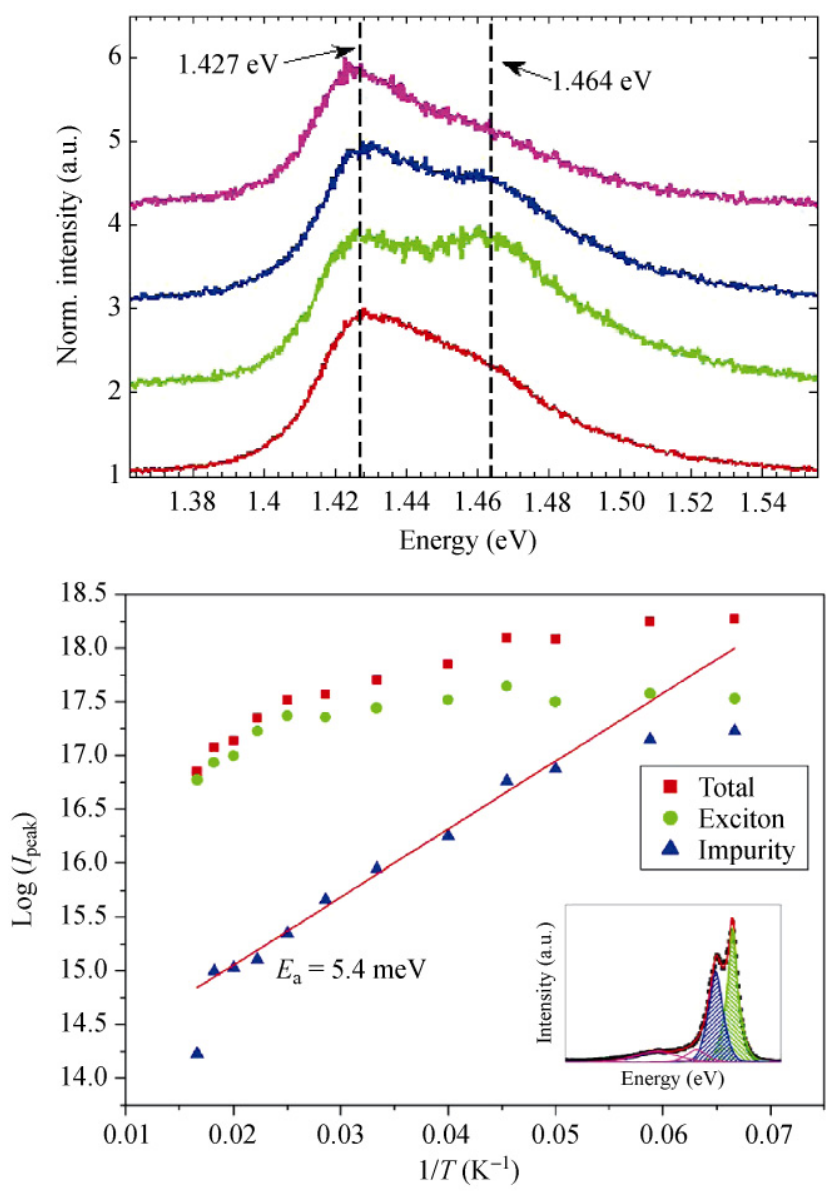

Figure 5 Top panel shows room temperature PL spectra of four different wires at a laser power of $30 \mathrm{~mW} / \mathrm{mm}^{2}$. The dotted lines indicate transitions between the conduction band and the first and second valence bands, respectively. In the bottom panel we have plotted the integrated intensity of total intensity, the exciton peak and the impurity peak as a function of inverse temperature 
In conclusion, we have measured PL and PLE spectra for pure wurtzite InP wires. From the spectra, the bandgap can be estimated to be about $1.493 \mathrm{eV}$. We also observe transitions involving a second valence band, present only in wurtzite $\mathrm{InP}$ and not in zinc blende InP. We find that the top of the valence band in wurtzite InP is split by about $40 \mathrm{meV}$, in agreement with other reports [12]. Temperature-dependent PL measurements reveal an impurity peak with a low activation energy, which is always present in the wires, as well as the existence of non-radiative recombination channels.

This work was performed within the Nanometer Structure Consortium at Lund University and was supported by the Swedish Research Council (VR), the Swedish Foundation for Strategic Research (SSF), and the Knut and Alice Wallenberg Foundation, and by the EU program AMON-RA (214814). This report is based on a project which was funded by E.ON AG as part of the E.ON International Research Initiative. Responsibility for the content of this publication lies with the authors.

\section{References}

[1] Mattila, M.; Hakkarainen, T.; Mulot, M; Lipsanen, H. Crystal-structure-dependent photoluminescence from InP nanowires. Nanotech 2006, 17, 1580-1583.

[2] Mishra, A.; Titova, L. V.; Hoang, T. B.; Jackson, H. E.; Smith, L. M.; Yarrison-Rice, J. M.; Kim, Y.; Joyce, H. J.; Gao, Q.; Tan, H. H.; Jagadish, C. Polarization and temperature dependence of photoluminescence from zincblende and wurtzite InP nanowires. Appl Phys Lett 2007, 91, 263104.

[3] Bao, J. M.; Bell, D. C.; Capasso, F.; Wagner, J. B.; Mårtensson, T.; Trägårdh, J.; Samuelson, L. Optical properties of rotationally twinned InP nanowire heterostructures. Nano Lett 2008, 8, 836-841.

[4] Algra, R.; Verheijen, M. A.; Borgström, M. T.; Feiner, L. F.; Immink, G.; van Enckevort, W. J. P.; Vlieg, E.; Bakkers, E. P.
A. M. Twinning superlattices in indium phosphide nanowires. Nature 2008, 456, 369-372.

[5] Paiman, S.; Gao, Q.; Tan, H. H.; Jagadish, C.; Pemasiri, K.; Montazeri, M.; Jackson, H. E.; Smith, L. M.; Yarrison-Rice, J. M.; Zhang, X.; Zou, J. The effect of V/III ratio and catalyst particle size on the crystal structure and optical properties of InP nanowires. Nanotech 2009, 20, 225606.

[6] Pemasiri, K.; Montazeri, M.; Gass, R.; Smith, L. M.; Jackson, H. E.; Yarrison-Rice, J.; Paiman, S.; Gao, Q.; Tan, H. H.; Jagadish, C.; Zhang, X.; Zou, J. Carrier Dynamics and Quantum Confinement in type II ZB-WZ InP Nanowire Homostructures. Nano Lett 2009, 9, 648-654.

[7] Caroff, P.; Dick, K. A.; Johansson, J.; Messing, M. E.; Deppert, K.; Samuelson, L. Nature Nanotech 2009, 4, 50-55.

[8] Glas, F.; Harmand, J. C.; Patriarche, G. Why does wurtzite form in nanowires of $\mathrm{III}-\mathrm{V}$ zinc blende semiconductors? Phys. Rev. Lett. 2007, 99, 146101.

[9] Dubrovskii, V. G.; Sibirev, N. V. Growth thermodynamics of nanowires and its application to polytypism of zinc blende III-V nanowires. Phys. Rev. B 2008, 77, 035414.

[10] Yamashita, T.; Sano, K.; Akiyama, T.; Nakamura, K.; Ito, T. Theoretical investigations on the formation of wurtzite segments in group III-V semiconductor nanowires. Appl. Surf. Sci. 2008, 254, 7668-7671.

[11] De, A.; Pryor, C. E. Predicted band structures of III-V semiconductors in the wurtzite phase. Phys. Rev. 2010, B81, 155210.

[12] Perera, S.; Pemasiri, K.; Fickenscher, M. A.; Jackson, H. E.; Smith, L; Yarrison-Rice, M. J.; Paiman, S.; Gao, Q.; Tan, H. H.; Jagadish, C. Probing valence band structure in wurtzite InP nanowires using excitation spectroscopy. Appl. Phys. Lett. 2010, 97, 023106.

[13] Magnusson, M. H.; Deppert, K.; Malm, J. O.; Bovin, J. O.; Samuelson, L. Size-selected gold nanoparticles by aerosol technology. Nanostructured Materials 1999, 12, 45-48.

[14] Ghandhi, S. K.; Parat, K. K. Shallow $\mathrm{N}^{+}$diffusion into InP by an open-tube diffusion technique. Appl. Phys. Lett. 1987, 50, 209-211.

[15] Bugajski, M.; Lewandowski, W. Concentration-dependent absorption and photoluminescence of n-type InP. J. Appl. Phys. 1985, 57, 521-530. 